\title{
Sustained and Accelerating Activity at Two Discrete Sites Generate Epileptiform Discharges in Slices of Piriform Cortex
}

\author{
Rezan Demir, ${ }^{1}$ Lewis B. Haberly, ${ }^{1,3}$ and Meyer B. Jackson ${ }^{1,2}$ \\ ${ }^{1}$ Center for Neuroscience, and Departments of ${ }^{2}$ Physiology and ${ }^{3}$ Anatomy, University of Wisconsin Medical School, \\ Madison Wisconsin 53706
}

\begin{abstract}
When near-threshold electrical stimulation is used to evoke epileptiform discharges in brain slices, a latent period of up to $150 \mathrm{msec}$ elapses before the discharge begins. During this period most neurons are silent, and abnormal electrical activity is difficult to detect with microelectrodes. A fundamental question about epileptiform activity concerns how synchronous discharges arise abruptly in a relatively quiescent slice. This issue was addressed here by using voltage imaging techniques to study epileptiform discharges in rat piriform cortex slices. These experiments revealed two distinct forms of electrical activity during the latent period. (1) A steeply increasing depolarization, referred to here as onset activity, has been described previously and occurs at the site of discharge onset. (2) A sustained depolarization that precedes onset activity, referred to here as plateau activity, has not been described previously.
\end{abstract}

Plateau and onset activity occurred in different subregions of the endopiriform nucleus (a region of high seizure susceptibility). When cobalt or kynurenic acid was applied focally to inhibit electrical activity at the site of plateau activity, discharges were blocked. However, application of these agents to other nearby sites (except the site of onset) failed to block discharges. Plateau activity represents a novel form of electrical activity that precedes and is necessary for epileptiform discharges. Discharges thus are generated in a sequential process by two spatially distinct neuronal circuits. The first circuit amplifies and sustains activity initiated by the stimulus, and the second generates the actual discharge in response to an excitatory drive from the first.

Key words: epilepsy; voltage imaging; piriform cortex; olfactory cortex; disinhibition; neural circuitry
In many in vitro models of epilepsy, electrical stimulation triggers discharges in which essentially every neuron is synchronously active. With stimulus currents only slightly above the threshold for triggering epileptiform discharges, a latent period elapses before the discharge begins. During this latent period most neurons are silent, and there is little abnormal electrical activity (Traub and Miles, 1991). The low level of latent-period activity makes it difficult to study the processes underlying epileptiform discharge generation. Computer simulations of epileptiform activity in hippocampus indicated that, during the latent period, abnormal activity is distributed sparsely over a large population of pyramidal cells (Traub et al., 1989; Traub and Miles, 1991). As a result of recurrent synaptic excitation the proportion of active neurons gradually builds until a critical level is reached and an epileptiform discharge begins. The idea that latent-period activity in the hippocampus is distributed sparsely receives support from imaging experiments, which have shown that discharges originate from diverse and variable locations throughout the CA2 and CA3 fields of hippocampal slices (Colom and Saggau, 1994).

Latent periods of up to $150 \mathrm{msec}$ have been observed to

Received Sept. 10, 1998; revised Nov. 30, 1998; accepted Dec. 1, 1998.

This research was supported by National Institutes of Health Grants NS19865 to L.B.H. and NS37212 to M.B.J. Additional funding was provided by the Wisconsin Alumni Research Foundation and the University of Wisconsin Medical School Research Committee. R.D. was supported by a fellowship from the American Association of University Women. We thank Drs. Phil Smith, Paul Rutecki, Matt Banks, Robert Pearce, and Tom Sutula for critical comments on this manuscript. We also thank Karen Manning for assistance in photographing histology section.

Correspondence should be addressed to Dr. Meyer Jackson, Department of Physiology, SMI 129, University of Wisconsin Medical School, 1300 University Avenue, Madison, WI 53706.

Copyright (๑) 1999 Society for Neuroscience $0270-6474 / 99 / 191294-13 \$ 05.00 / 0$ precede epileptiform discharges in slices of rat piriform cortex (PC) (Hoffman and Haberly, 1989, 1996; Demir et al., 1998). In contrast to the hippocampal slice, discharges in PC slices begin at a well defined location that can include the dorsal-most part of endopiriform nucleus (En) (Hoffman and Haberly, 1991, 1993, 1996) and adjoining layer VI of neocortex (Demir et al., 1998). This localization of onset gives the PC slice a potential advantage in studying the genesis of epileptiform activity. In the dorsal-most region of En an accelerating build-up in activity during the latent period was observed that resembles that seen in hippocampus (Hoffman and Haberly, 1993). Pharmacological, physiological, and anatomical analysis in the En revealed recurrent excitatory synaptic activity within the dominant multipolar cell population, suggesting that the hypothesis of synaptically mediated positive feedback, developed as a mechanism for the latent period in hippocampus (Ayala et al., 1973; Johnston and Brown, 1986; Traub et al., 1989; Traub and Miles, 1991), also may be applicable to the PC (Hoffman and Haberly, 1993).

Using voltage imaging techniques (Grinvald et al., 1988; Wu and Cohen, 1993), we examined the spatiotemporal pattern of electrical activity in slices of PC and observed the accelerating build-up of activity at the onset site described previously (Hoffman and Haberly, 1993; Demir et al., 1998). In addition, a second form of abnormal activity was observed during the latent period, which has not been described previously. This activity has distinct temporal properties and is restricted to a zone at the boundary of En with deep layer III of PC. This region showed very little spatial overlap with the onset site in dorsal-most En and vicinity. Local application of synaptic inhibitors suggested that this newly described activity plays an essential role in the generation of epileptiform discharges in PC slices. 
A preliminary account of these findings has been published previously (Demir et al., 1997).

\section{MATERIALS AND METHODS}

Piriform cortex slices. Slices through PC and En of adult male rats were cut at a thickness of $350 \mu \mathrm{m}$ in a near-coronal plane perpendicular to the brain surface (Hoffman and Haberly 1991, 1993; Demir et al., 1998). The physiological solution for slice preparation and recording was (in $\mathrm{mm}$ ): $124 \mathrm{NaCl}, 5 \mathrm{KCl}, 26 \mathrm{NaHCO}_{3}, 1.2 \mathrm{KH}_{2} \mathrm{PO}_{4}, 2.4 \mathrm{CaCl}_{2}, 1.3 \mathrm{MgSO}_{4}$, and 10 glucose bubbled with $95 \% \mathrm{O}_{2} / 5 \% \mathrm{CO}_{2}$. This solution with elevated $\mathrm{K}^{+}, \mathrm{Ca}^{2+}$, and $\mathrm{Mg}^{2+}$ has been used in previous studies with this slice model. In normal $\mathrm{K}^{+}$and $\mathrm{Ca}^{2+}$ similar activity is observed (Hoffman and Haberly, 1991). Recordings were made at $32 \pm 2^{\circ} \mathrm{C}$. A sketch of a PC slice is shown in Figure 1 to illustrate the features relevant to this study. Important landmarks present in all slices are the three layers of the PC, the underlying En, and the external capsule, which separates PC from caudate putamen. Most of the slices studied here were taken from a portion of PC corresponding to intermediate slices of Demir et al. (1998) [anteroposterior, -1.80 to $-0.70 \mathrm{~mm}$ (Paxinos and Watson, 1986)]. This stereotaxic level encompasses the boundary between agranular insula and anterior perirhinal cortex [terminology of Cechetto and Sapir (1987), Burwell et al. (1995), and McIntyre et al. (1996)], so that PC in these slices is bounded laterally by the junctional region between these two cytoarchitecturally distinct forms of neocortex. A small portion of the claustrum was present in the more rostral slices (Fig. 1).

Epileptiform activity. Two methods were used to obtain epileptiform activity in brain slices. The first method, termed induction, involved a transient period of bursting to induce a long-term, NMDA receptordependent increase in excitability (Hoffman and Haberly, 1989; Stasheff et al., 1989). Slices were bathed for $30-90 \mathrm{~min}$ at $34^{\circ} \mathrm{C}$ in low-chloride physiological saline with the composition given above, but with $93 \%$ of the $\mathrm{Cl}^{-}$replaced by isethionate. Slices then were returned to control saline for imaging experiments. Once induced in this manner, slices continued to show epileptiform activity for the duration of the experiments (up to $7 \mathrm{hr}$ ). The second method, termed disinhibition, entailed bath application of the $\mathrm{GABA}_{\mathrm{A}}$ receptor antagonist bicuculline methiodide (5-10 $\mu \mathrm{M}$; obtained from Sigma, St. Louis, MO), and this drug was present during the actual recordings. Epileptiform activity elicited by these two methods exhibited subtle differences in temporal and spatial characteristics, implying differences in the underlying mechanism of discharge generation (Demir et al., 1998).

Epileptiform discharges were evoked by current pulses applied through a saline-filled glass pipette $(20-50 \mu \mathrm{m}$ tip diameter). In experiments involving local application of $\mathrm{CoCl}_{2}$ or kynurenic acid, epileptiform discharges were elicited by stimulus currents $\sim 10 \%$ above discharge threshold to avoid subthreshold responses. Because the threshold current for discharge generation varied in the course of long experiments (3-5 $\mathrm{hr}$ ), the threshold was checked regularly.

Voltage imaging. Slices were stained for 30-45 min with the voltagesensitive fluorescent dye RH414 (Molecular Probes, Eugene, OR) at a concentration of $200 \mu \mathrm{M}$. Slices then were used immediately for imaging experiments. Imaging was performed with a Reichert Jung Diastar fluorescence microscope (Leica, Deerfield, IL) equipped with a 475-565 $\mathrm{nm}$ bandpass excitation filter, a $570 \mathrm{~nm}$ dichroic mirror, and a $610 \mathrm{~nm}$ longpass emission filter. A $100 \mathrm{~W}$ tungsten-halogen bulb provided illumination. Fluorescent light was focused onto a photodiode fiber optic device (Chien and Pine, 1991) with 464 hexagonally arranged detectors. Most fluorescence recordings were made with a Zeiss Fluar $5 \times$ objective (numerical aperture, 0.25), which produced an image with $144 \mu \mathrm{m}$ between photodiode fields. Signals were amplified as described previously (Wu and Cohen, 1993; Jackson and Scharfman, 1996; Demir et al., 1998 ), with two stages of amplification producing an output of $0.2 \mathrm{~V} / \mathrm{pA}$ of photocurrent. Photocurrent was low-pass-filtered at $500 \mathrm{~Hz}$ and highpass-filtered with a $500 \mathrm{msec}$ time constant. All fluorescence traces shown were normalized by the resting light intensity of each detector and filtered digitally with a three point binomial filter. Displayed fluorescence traces were averages of signals recorded by four or five neighboring detectors taken from single trials. Previous work has shown that RH414 fluorescence corresponds well with extracellularly recorded potentials in PC slices and is altered appropriately by various pharmacological manipulations (Demir et al., 1998).

Data acquisition and analysis. Images were digitized with a DAP 3200e/214 analog-to-digital converter (Microstar, Bellevue, WA) running on a Pentium computer. The computer program NeuroPlex (OptImaging, Fairfield, CT) was used for data acquisition and most analysis. This

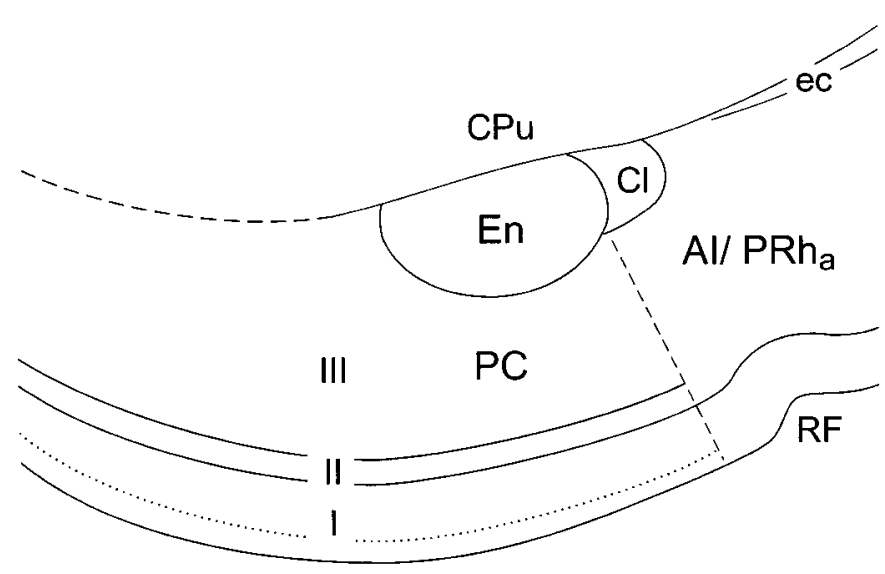

Figure 1. Sketch of a piriform cortex $(P C)$ slice. Roman numerals indicate the layers of PC. En, Endopiriform nucleus; $e c$, external capsule; $A I$, agranular insula; $P R h_{\mathrm{a}}$, anterior perirhinal cortex; $R F$, rhinal fissure; $C P u$, caudate putamen; $\mathrm{Cl}$, claustrum.

program runs under IDL (Research Systems, Boulder, CO) and digitizes the fluorescence of all 464 channels at a rate of $0.94 \mathrm{msec} / \mathrm{frame}$. To identify the site of discharge onset, we first determined the earliest time point at which fluorescence reaches 50 or $70 \%$ of its maximum amplitude. Contours then were drawn around these detectors at which fluorescence first reached this level by using an additional program written in IDL. Then these contours were overlaid on a video image of the slice with another IDL program. This method of identifying the site of onset gave the same results as a manual method that was based on visual inspection of individual traces (Demir et al., 1998). Plateau activity (see Results) was generally $25 \%$ of the maximal discharge amplitude, so the $50-70 \%$ cutoff excluded plateau activity from the site-of-onset contour. The low amplitude of plateau activity made automated methods of analysis more difficult. Plateau activity was identified by a visual inspection of individual traces on the basis of whether it was clearly visible above background noise before discharge onset. Contours then were drawn manually around those detectors.

Local drug application. A micropipette with a 3-4 $\mu \mathrm{m}$ tip (inner diameter) was filled with $10 \mathrm{~mm} \mathrm{CoCl}$ or $5 \mathrm{~mm}$ kynurenic acid (Aldrich, Milwaukee, WI) in $0.9 \% \mathrm{NaCl}$ (Hoffman and Haberly, 1996). Solution was ejected with $0.5 \mathrm{sec}, 20-25$ psi pressure pulses from a Picospritzer (General Valve, Fairfield, NJ). In control slices the $\mathrm{CoCl}_{2}$ reduced local responses at the site of application by $20 \pm 4 \%(n=9)$. Before every $\mathrm{CoCl}_{2}$ or kynurenic acid application, three epileptiform responses were recorded to confirm that the discharge latency was stable. $\mathrm{CoCl}_{2}$ or kynurenic acid was applied three times at each site in each experiment. The inclusion of $0.1 \mathrm{mg} / \mathrm{ml}$ fluorescein in the drug solution allowed for verification of fluid ejection in every experiment and provided an indication of the extent of drug localization. Fluorescein fluorescence was viewed with a different optical filter set from that used for voltage imaging (450-490 nm emission filter, $490 \mathrm{~nm}$ dichroic mirror, 510-560 $\mathrm{nm}$ bandpass excitation filter). Because of the different spectral properties of RH414, fluorescein did not interfere with voltage imaging. The localized application of drug with this technique also was evaluated in experiments showing that graded responses were reduced only within $500 \mu \mathrm{m}$ of the site of application. Furthermore, discharges could be blocked or not blocked, depending on the site of drug application (see Results); moving the application pipette $<400 \mu \mathrm{m}$ generally gave qualitatively different results.

Histology. After each experiment the slices were fixed in $4 \%$ paraformaldehyde, sectioned at $60 \mu \mathrm{m}$ with a freezing microtome, and stained with cresyl violet for histological analysis.

\section{RESULTS}

\section{Spatiotemporal patterns of control and epileptiform activity}

In control slices, electrical stimulation in layer $\mathrm{Ib}$ of the $\mathrm{PC}$ evoked a rapidly decaying local response (see Fig. 2A1). Response amplitudes in control slices decreased with distance from 


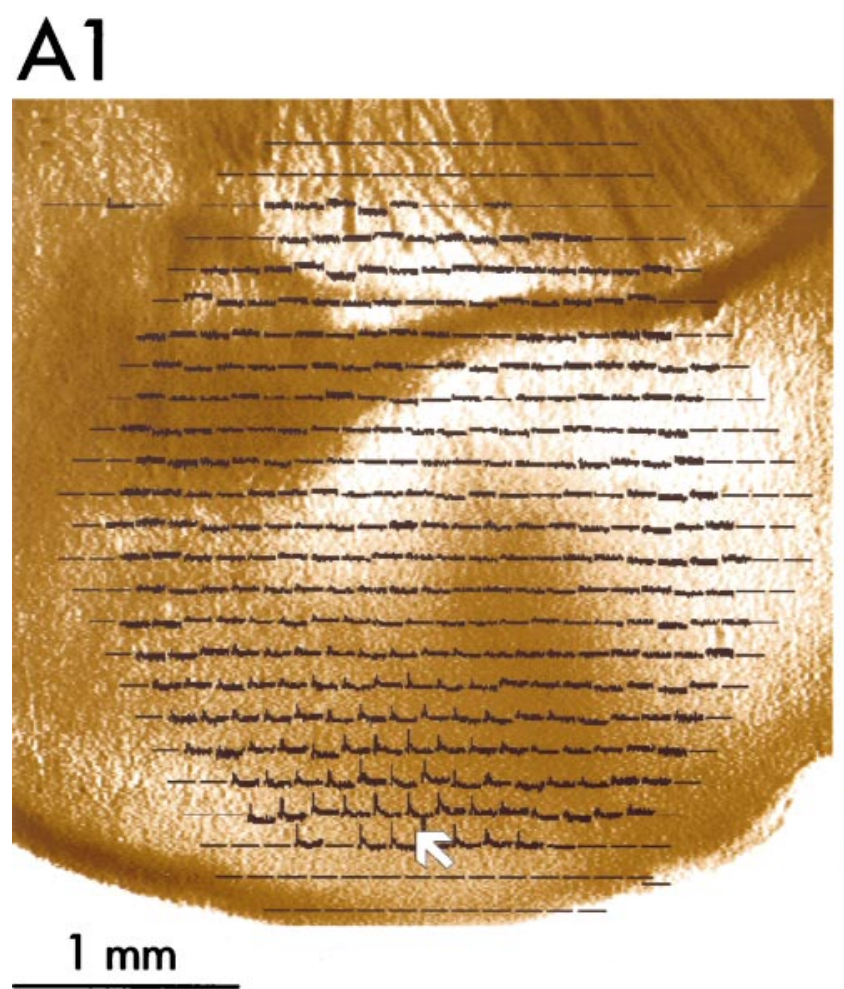

A2

B1
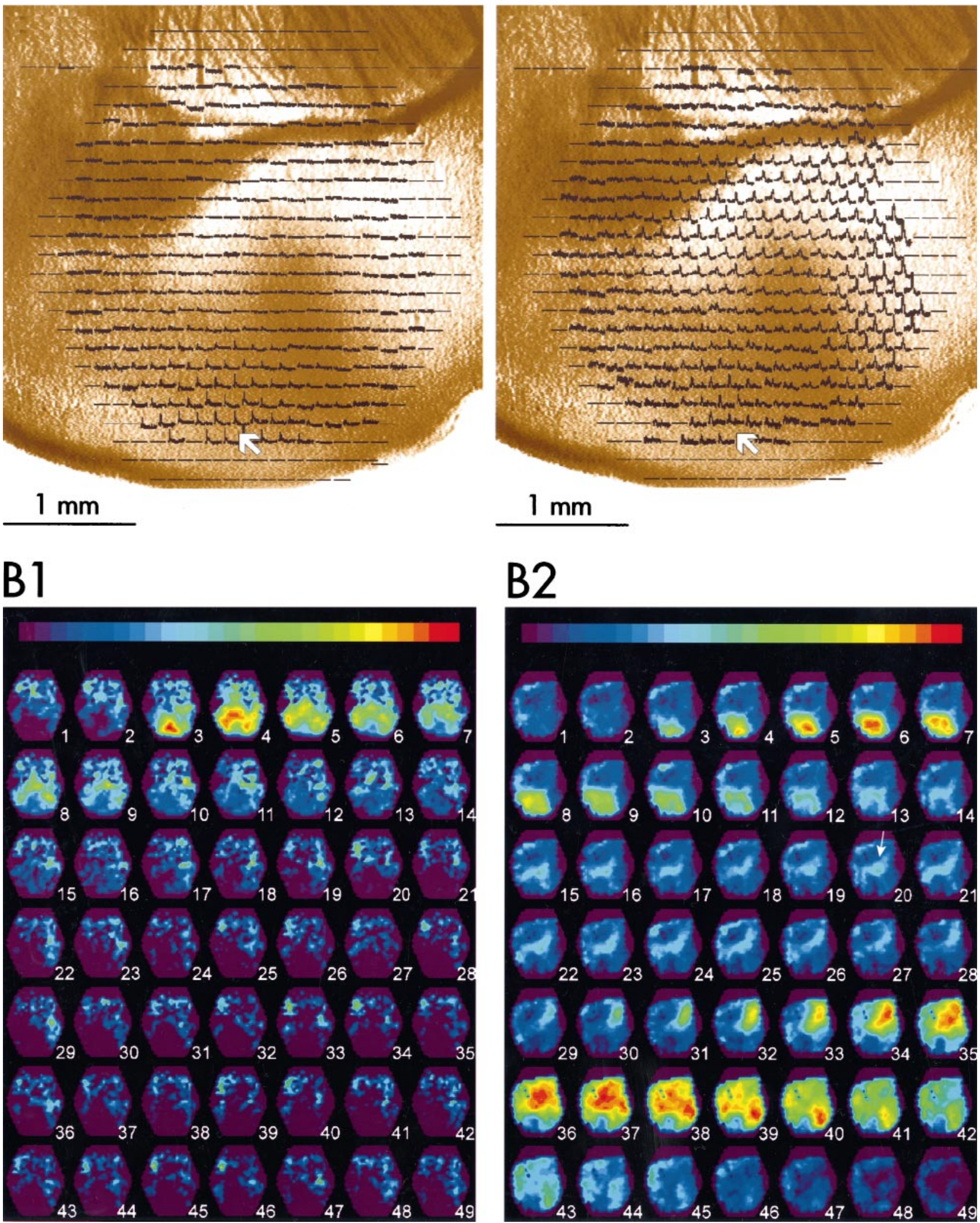

B2

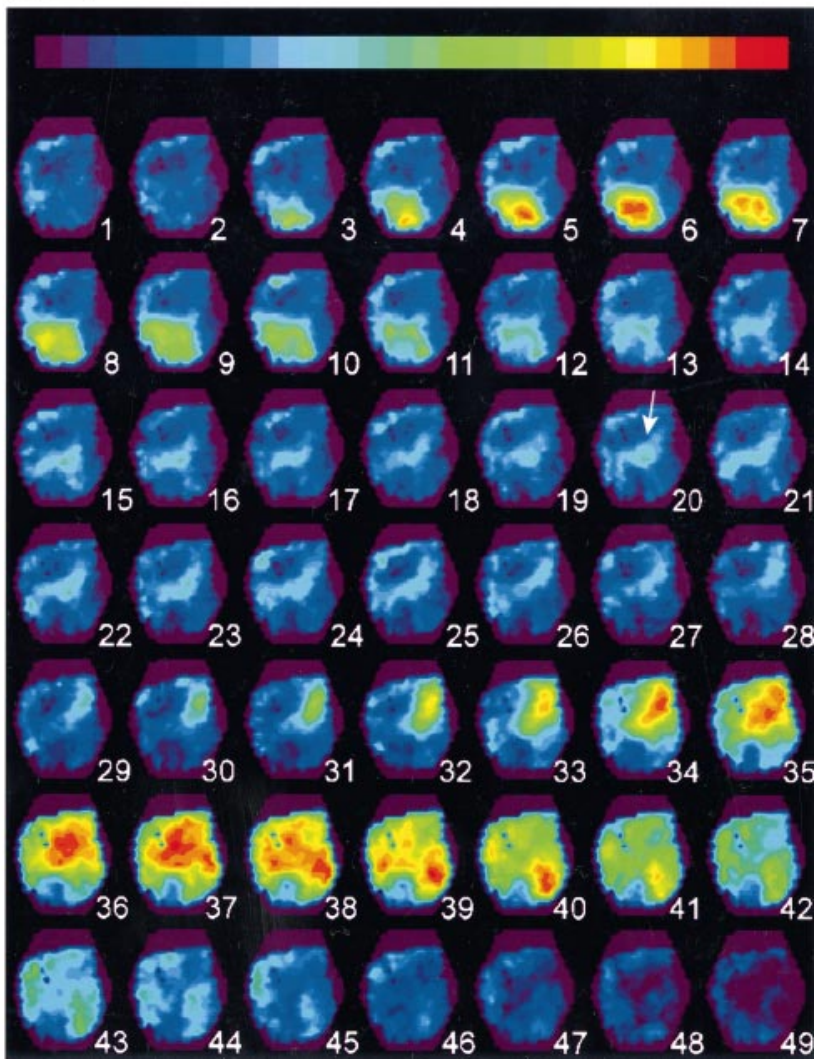

Figure 2. Spread of electrical activity in PC slices. $A$, Overlays of fluorescence traces on video images show the spread of electrical activity (later figures show expanded views of selected traces). $A 1$, In a control slice responses were largest near the stimulus electrode (stimulus at $100 \mu \mathrm{A}, 200 \mu$ sec). $A 2$, In a disinhibited slice (with $10 \mu \mathrm{M}$ bicuculline methiodide) a weaker stimulus ( $30 \mu \mathrm{A}, 200 \mu \mathrm{sec}$ ) evoked epileptiform activity that was evident through most of the slice. Stimulation sites in layer Ib are indicated by arrows. Trace durations are $450 \mathrm{msec}$, with the stimulus applied 28 msec after the start of the trace. $B$, Sequences of intensity-coded color maps show the temporal pattern of spread for the overlays in $A$. The code (Figure legend continues) 


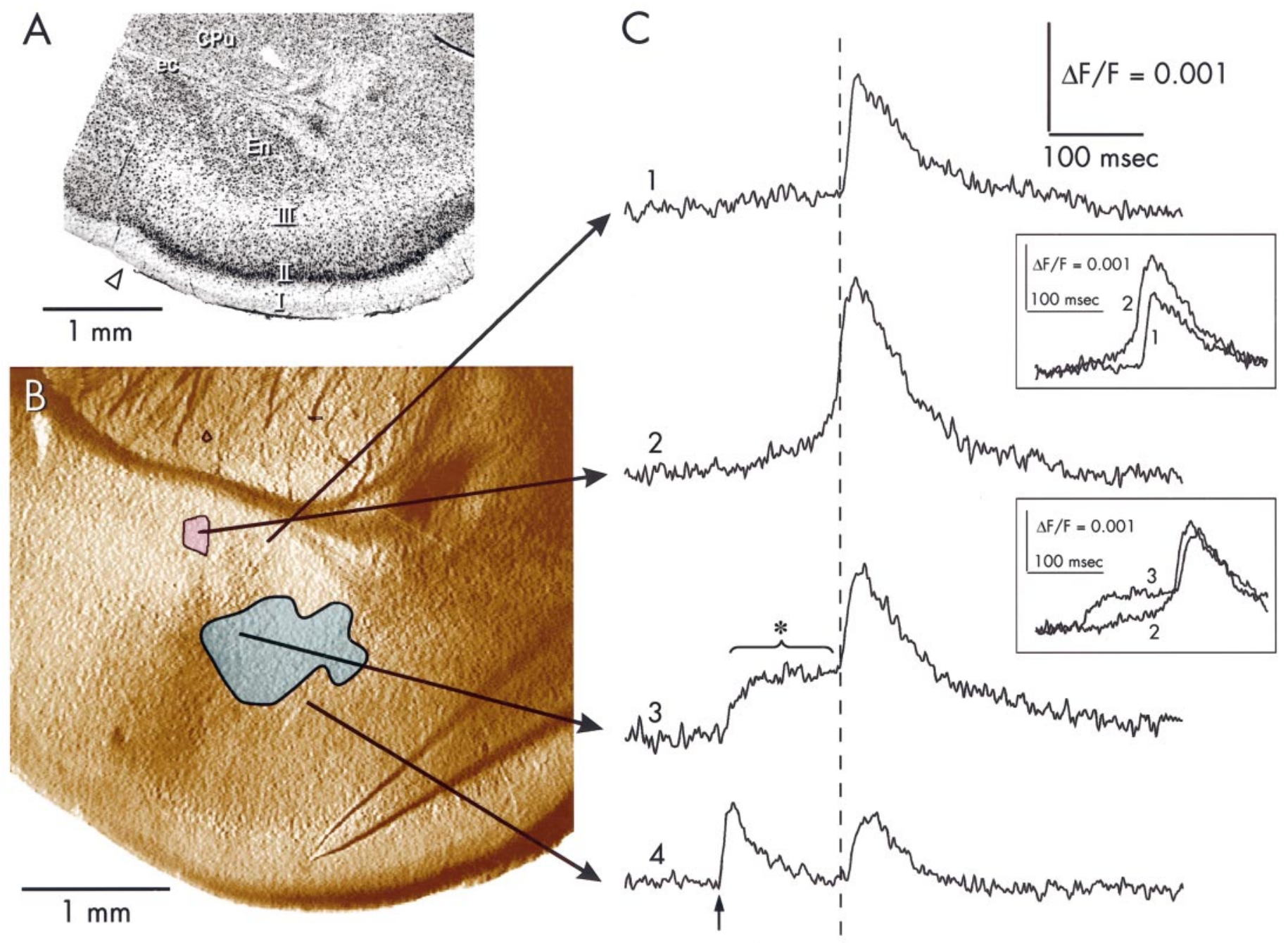

Figure 3. Voltage-sensitive dye fluorescence at selected locations during an epileptiform discharge. A, A Nissl-stained slice was prepared after the imaging experiment to identify anatomical structures (labels as in Fig. 1). The arrowhead marks the border between PC and adjacent neocortex. $B$, A video image of the same slice taken during recordings shows the site of onset of an epileptiform discharge (pink) and the site of plateau activity (blue). Epileptiform discharges were evoked by a $132 \mu \mathrm{A}, 200 \mu \mathrm{sec}$ stimulus, with the electrode visible in layer Ib. $C$, Fluorescence traces from the indicated sites. Trace 1 was taken from the central part of the En in which activity started abruptly after onset activity and plateau activity at other sites. Trace 2 was taken from the site of onset in dorsal-most En and shows ramp-like onset activity leading to an epileptiform discharge. A comparison of latencies at the different sites with the aid of the dashed line shows that the discharge appeared at this location first. Trace 3 shows plateau activity (indicated by a starred bracket). Trace 4 from a site in layer III outside the plateau activity region shows that the local response decayed before the discharge began. The arrow indicates the time of stimulus. The top inset in $C$ shows traces 1 and 2 superimposed to emphasize the ramp-like character of onset activity. This contrasts with the abrupt emergence of the discharge at a longer latency at a nearby location. The bottom inset shows traces 2 and 3 superimposed to illustrate that plateau activity precedes the ramp-like build-up at the site of onset by $10-20 \mathrm{msec}$. This superposition also highlights the differing time courses of plateau activity and onset activity.

the site of stimulus and were very small in the deep regions (e.g., $E n$ in Fig. 1). Thus the spread of evoked signals in control slices is restricted both spatially and temporally (Demir et al., 1998). In contrast, in slices in which epileptiform activity was elicited either by induction or disinhibition, electrical activity spread through most of the piriform region and into adjacent neocortex. Figure
$2 A 2$ illustrates this spread in a disinhibited slice. Slices showing epileptiform activity still produced the brief local response to electrical stimulation seen in control slices (Fig. 2B2, frames 3-12) regardless of whether the stimulus current was sub- or suprathreshold for epileptiform discharges. When the stimulus current exceeded threshold, an epileptiform discharge emerged

for fluorescence intensity is indicated by the scale bar at the top (increasing depolarization from purple to red). Sequentially numbered frames represent time points at $5.7 \mathrm{msec}$ intervals. B1, A control slice shows a local response immediately after the stimulus (red spot in frame 3 ), which decays to the prestimulus background level by frame 12. B2, In a disinhibited slice, the local response displays an approximately similar time course. An epileptiform discharge began in the dorsal-most portion of the En in frame 30. The discharge intensified and spread through the overlying PC and neighboring neocortex in subsequent frames. During the latent period a persistent light blue spot in the middle of frames 12-28 (indicated by a white arrow in frame 20) shows activity not evident in the prestimulus frames. This represents plateau activity displayed more clearly in subsequent figures. Note that the later frames in $B 1$ (after frame 15) are darker than frames 1 and 2 preceding the stimulus. This reflects slow inhibition after the rapid excitatory response. 

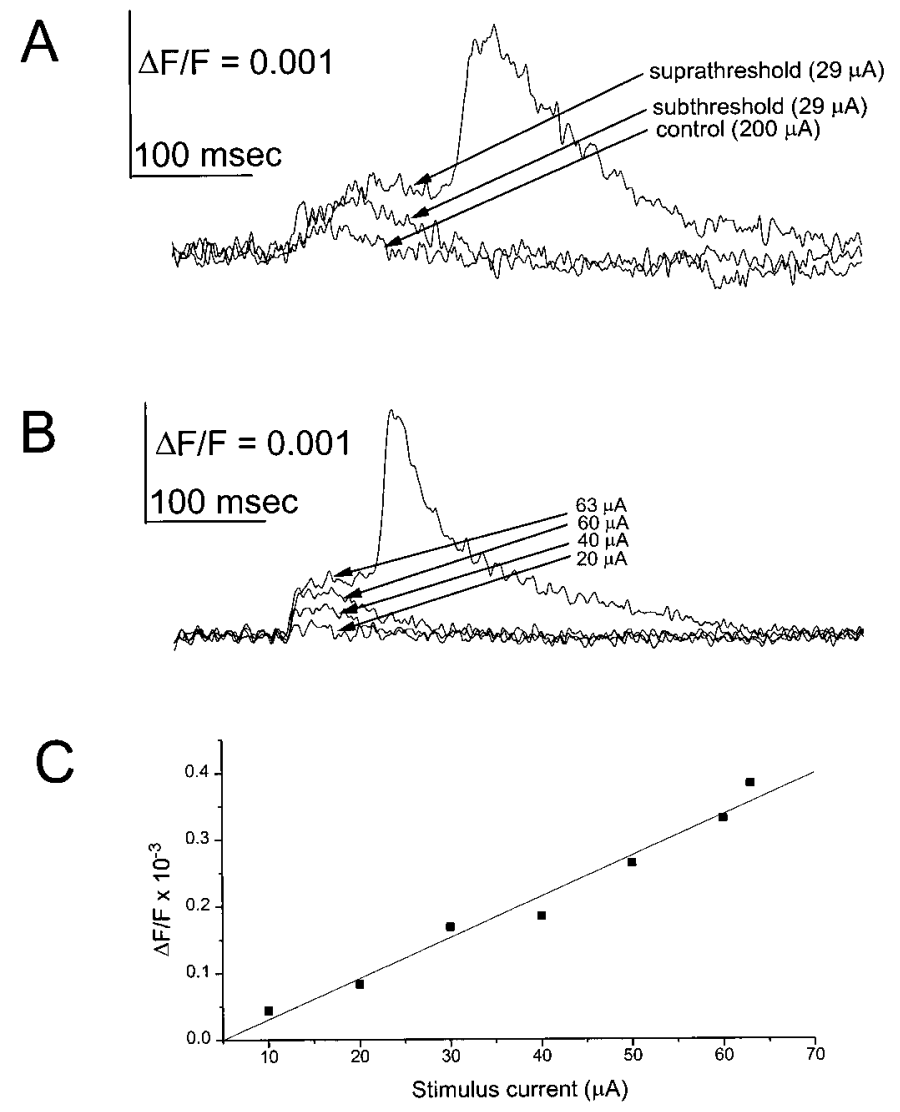

Figure 4. Responses at the site of plateau activity. $A$, Fluorescence signals were taken from a location near the border between the En and layer III at a location within the region shaded blue in Figure $3 B$. Before the addition of bicuculline methiodide to the bath, a $200 \mu \mathrm{A}, 200 \mu \mathrm{sec}$ stimulus applied in layer Ib (site is similar to that shown in Fig. 2B) evoked a small, rapidly decaying control response (bottom trace). After bath application of $5 \mu \mathrm{M}$ bicuculline methiodide, a threshold stimulus current $(29 \mu \mathrm{A})$ was used to evoke subthreshold and suprathreshold epileptiform responses with equal probability. In suprathreshold responses the plateau activity had a slightly higher level than the peak of the subthreshold response. $B$, A series of subthreshold responses to stimuli of increasing strengths is shown at the site of plateau activity in an induced slice. $C$, A plot of response versus stimulus strength at the site of plateau activity shows graded responses to increasing stimulus current. Responses plotted are to subthreshold stimulus currents, except the final point, which gives the amplitude of plateau activity in a suprathreshold response. This plot was made from the same series of responses used in $B$. Two other plots were examined and showed similar behavior.

$\sim 100$ msec after the local response from a distinct location at the dorsal-most portion of the En near its boundary with claustrum and neocortex (Fig. 2B2, frame 30). After onset, the discharge spread to the overlying layers of PC and to deep and superficial layers of the neighboring neocortex (Fig. 2B2, frames 30-43). The site of discharge onset (as defined by detectors in which the fluorescence first reached $50-70 \%$ of maximum; see Materials and Methods) included the dorsal-most part of the En (Hoffman and Haberly, 1993) and adjoining ventral-most part of agranular insula, rostrally, and anterior perirhinal cortex, caudally, in agreement with previous imaging studies (Demir et al., 1998).

Epileptiform discharges throughout the PC, adjacent neocortical areas, and most of the En exhibited a latent period during which most of the slice appeared quiescent (Fig. 2B2, frames 13-30). However, a restricted region was found in which electrical activity was sustained at a substantial level throughout the entire interval between stimulus and discharge onset. This can be seen in frames $13-30$ of Figure 2B2, where a patch in the middle of the display area remained light blue throughout the latent period (as indicated by an arrow in frame 20). This suggests that, as the local response dies out, a part of the slice remains active. Fluorescence traces confirmed that electrical activity is maintained at this location during the entire latent period (Fig. 3C, trace 3, activity indicated by the starred bracket). With near-threshold stimulation the fluorescence intensity at this location remained at an approximately constant fraction of $25 \pm 2 \%(n=12)$ and $24 \pm 2 \%(n=$ 21) of the fluorescence intensity observed at the discharge peak in disinhibited and induced slices, respectively (see Fig. 5 below). This indicates that this site was a focus of sustained depolarization before the discharge onset. Because this activity exhibits an approximately constant amplitude throughout the latent period, it will be referred to here as plateau activity. This term distinguishes it from the steeply rising depolarization (Fig. 3C, trace 2) described previously at the site of discharge onset (Hoffman and Haberly, 1993; Demir et al., 1998), which will be referred to here as onset activity.

\section{Localization of plateau and onset activity}

To identify the sites of plateau and onset activity, we conducted histological examinations after imaging experiments. Plateau activity was confined to a part of the En and adjacent deep layer III of PC (Fig. 3A,B). As noted above, the site of discharge onset resides in the dorsal-most part of the En and layer VI of the adjoining neocortex. [Note that the site of onset in Fig. 3 did not extend into layer VI of the adjoining neocortex, because this experiment was performed in a slice at a rostrocaudal level at which onset occurs only in En (for details, see Demir et al., 1998).] Contours around the region containing plateau activity generally did not include the site of onset. Figure $3 B$ shows that the blue-shaded plateau region and the pink-shaded onset region do not overlap. In only a few experiments was a small amount of overlap seen. Traces from the site of discharge onset showed no evidence of plateau activity (Fig. 3C, trace 2). Instead, this location showed ramp-like onset activity, as described previously (Hoffman and Haberly, 1993). Traces from other locations such as the deep central part of the En (Fig. 3C, trace 1), layer III (Fig. $3 C$, trace 4), and adjoining neocortex (data not shown) also showed no plateau activity. At these sites epileptiform discharges arose abruptly from a flat baseline. Trace 4 of Figure $3 C$ was taken just outside the region showing plateau activity, from a location close enough to the stimulus site to show a large local response. Activity at this site decayed to baseline before discharge onset.

The insets of Figure $3 C$ show superimposed traces to illustrate these temporal relationships more clearly. Superposition of traces from the site of onset (2) and another site (1) in the En shows that the ramp-like build-up of onset activity represents a substantial departure from baseline well before the discharge begins (top inset, Fig. $3 C$ ). Superposition of traces from the site of onset (2) and the site of plateau activity (3) shows that fluorescence signals associated with plateau activity are larger than fluorescence signals associated with onset activity until immediately before onset (bottom inset, Fig. 3C). Furthermore, the superposition of these two traces emphasizes the fact that plateau activity precedes the earliest appearance of onset activity by $\sim 20 \mathrm{msec}$ (range, 10-50 $\mathrm{msec}$ ). Superposition of these traces also makes it clear that, although plateau activity preceded onset activity, the discharge at the site of plateau activity always followed the discharge at the site 


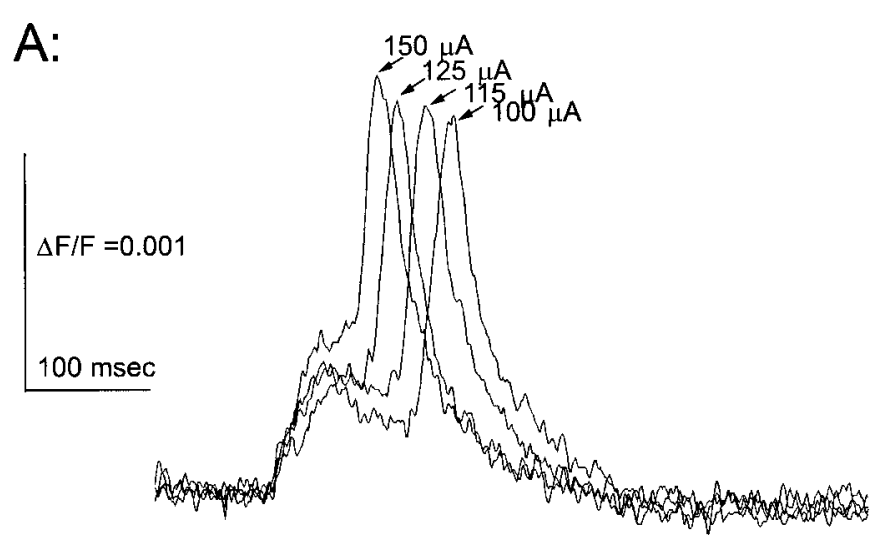

\section{$B$ : induced}

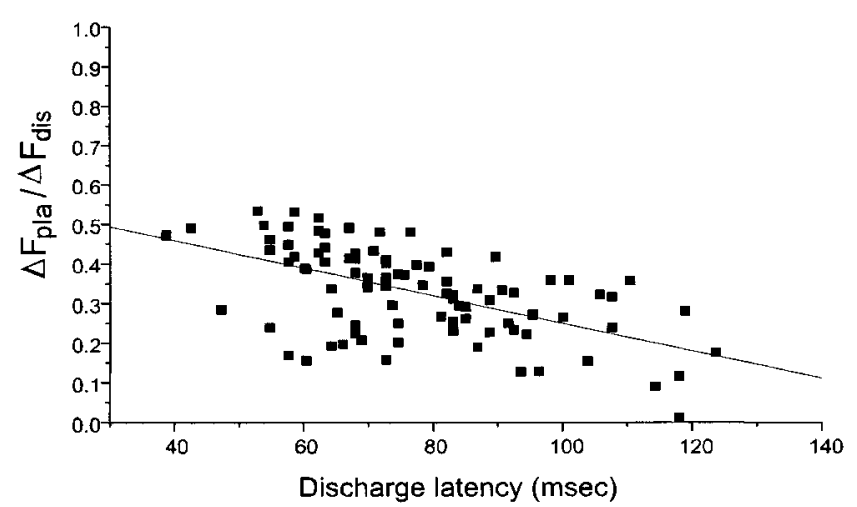

\section{C: disinhibited}

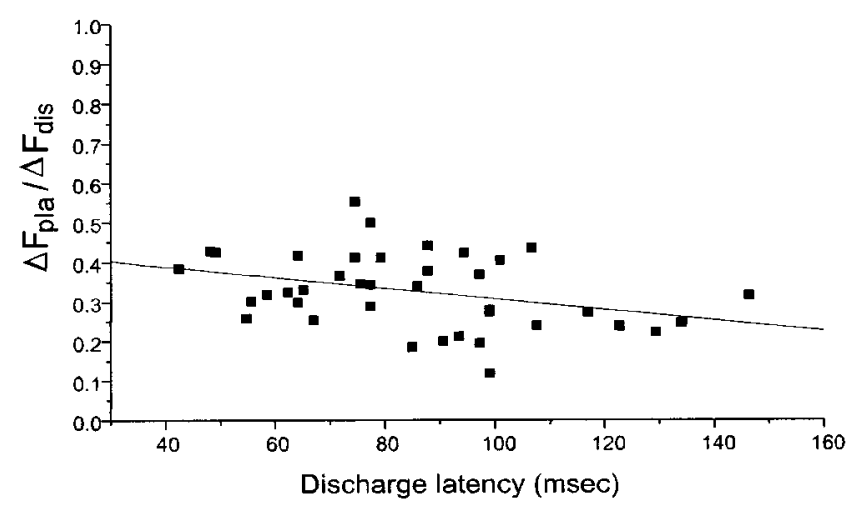

Figure 5. A, Superimposed traces from the site of plateau activity for four suprathreshold stimuli. Increasing the stimulus strength produced a decrease in discharge latency concomitant with an increase in the amplitude of plateau activity. $B, C$, The peak change in fluorescence associated with the plateau event $\left(\Delta F_{p l a}\right)$ was normalized to the peak fluorescence change of the epileptiform discharge $\left(\Delta F_{\text {dis }}\right)$ and plotted versus discharge latency. Each individual trace gave one point for this plot, but because stimuli of different strengths could be used on one slice, many points could be obtained per experiment. $B$, Data were pooled from eight induced slices. $C$, Data were pooled from seven disinhibited slices. The stimulus strength was varied to obtain the range of latencies plotted. Linear regression showed a statistically significant correlation with $r=-0.57$ $\left(p<10^{-8}\right)$ in $B$ and $r=-0.35(p<0.05)$ in $C$. of onset $(n>50)$. It is important to note that the only forms of abnormal electrical activity seen during the latent period before discharge onset were onset and plateau activity at their characteristic locations. No other form of electrical activity was evident elsewhere during this time other than the decaying local response that is seen in control slices as well.

\section{Properties of plateau activity}

Regardless of whether epileptiform activity was obtained by induction in a low- $\mathrm{Cl}^{-}$medium $(n=36)$ or by disinhibition with the $\mathrm{GABA}_{\mathrm{A}}$ receptor antagonist bicuculline $(n=16$; see Materials and Methods), plateau activity was seen at essentially the same location. Under either epileptiform condition and for diverse stimulation sites within PC slices taken from levels along the entire anterior-posterior axis, plateau activity occurred at approximately the same site illustrated in Figure $3 B$. Thus, the region identified by the presence of plateau activity possesses special properties that enable it to sustain electrical activity under two different in vitro models of epilepsy.

Plateau activity differed from the graded responses observed in the same region in control slices. Figure $4 A$ shows a control response (in normal saline) from a site subsequently shown to have plateau activity. This trace is superimposed with a subthreshold graded response and suprathreshold epileptiform response recorded from the same site after disinhibition with bicuculline methiodide. The control response at this site was small and decayed rapidly. Note that a strong stimulus current $(200 \mu \mathrm{A})$ evoked a control response that was much smaller than the subthreshold response evoked by a weak stimulus current $(29 \mu \mathrm{A})$ after disinhibition (Fig. 4A). Thus, even without epileptiform discharges, subthreshold responses revealed a large increase in excitability as a result of disrupting inhibition. Like control responses, subthreshold responses under epileptiform conditions were graded with stimulus intensity. A series of subthreshold responses is shown in Figure 4B, and a plot of response amplitude versus stimulus current shows that the peak subthreshold response at the site of plateau activity increased approximately linearly with stimulus intensity (Fig. $4 C ; n=3$ ). This demonstrates that under epileptiform conditions subthreshold responses are graded like control responses, only much larger. This graded property stands in striking contrast with the all-or-none character of epileptiform discharges (Figs. 4B, 5A).

A comparison of the amplitude and time course of control responses in normal saline and subthreshold responses under epileptiform conditions suggests that subthreshold responses may have a new process added to the control response. From this perspective, epileptiform conditions could enhance the di- and polysynaptic potentials that have been demonstrated previously in the deep cells of the PC (Tseng and Haberly, 1989a). Subthreshold responses at the site of plateau activity had slightly smaller peak amplitudes than plateau activity associated with epileptiform discharges (Fig. 4A). Further, subthreshold responses never had a plateau-like appearance but decayed smoothly back to baseline. Depolarization with a plateau-like time course was never seen in isolation from an epileptiform discharge. Additional experiments described below show that when onset activity was blocked, responses from the site of plateau activity still had a much higher amplitude than control responses but no longer had a plateau-like appearance, because they decayed smoothly to baseline. This introduces an important point that synaptic input from the site of onset helps to maintain the depolarization at the 

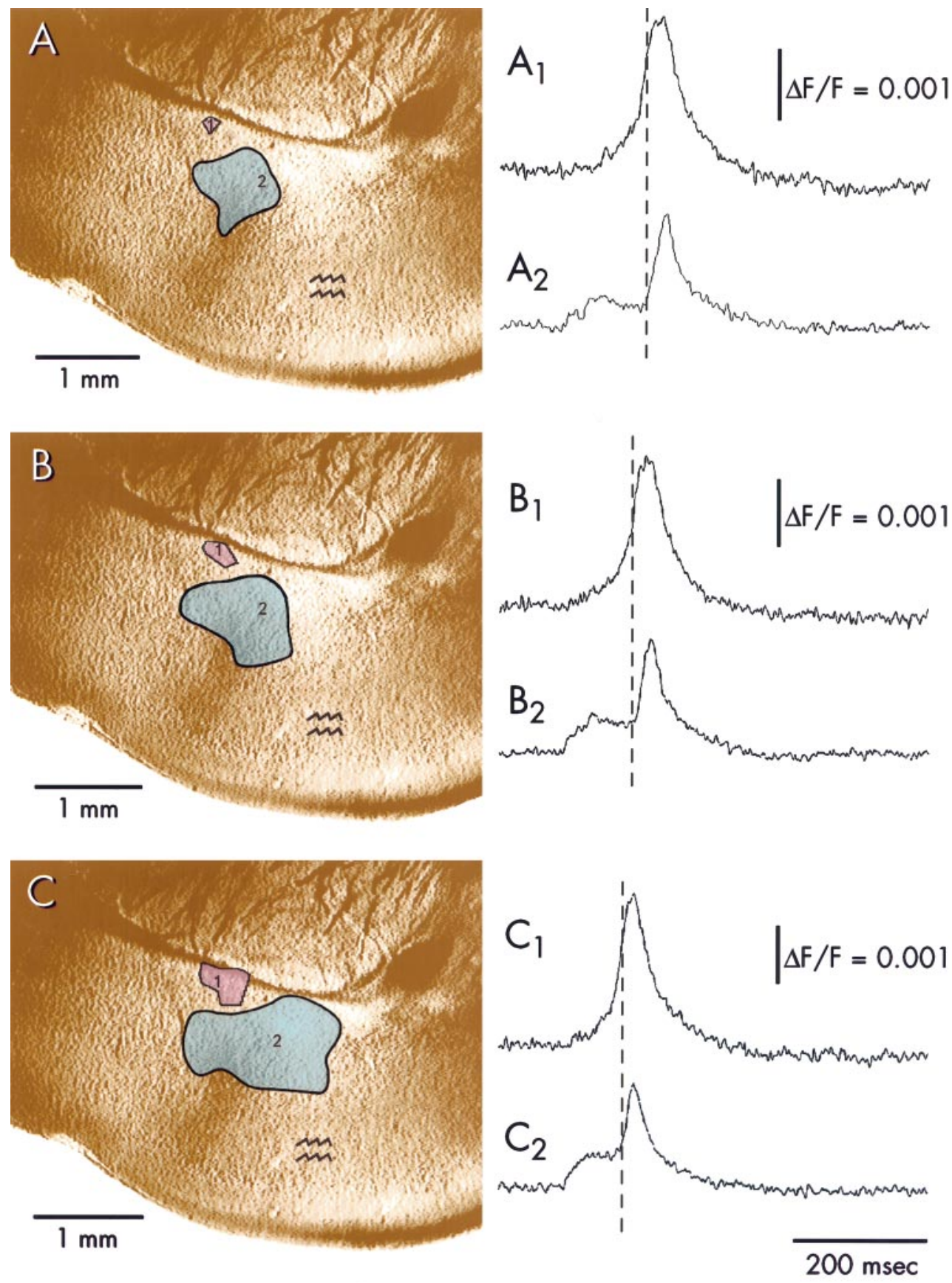

Figure 6. Variations in size of sites of onset and plateau activity with increasing stimulus current. Traces are taken from the regions shown. As in Figure 3, the site of onset is shaded pink (traces $A_{1}, B_{1}$, and $C_{1}$ ) and the site of plateau activity is shaded blue (traces $A_{2}, B_{2}$, and $\left.C_{2}\right) . A$, With a stimulus current at the threshold for epileptiform discharge generation $(100 \mu \mathrm{A})$, the sizes of both onset and plateau regions were smallest. Increasing stimulus current to $115 \mu \mathrm{A}$ $(B)$ and $125 \mu \mathrm{A}(C)$ increased the sizes of both regions. The traces show a shortening of the latent period and an increase in amplitude of plateau activity as the stimulus current was increased (see Figs. 4, 5). The site of stimulus is marked by the double wavy lines. Vertical dashed lines show that the discharge appeared at the site of onset first.

site of plateau activity. The relationship between activity at these two sites will receive further consideration in Discussion.

\section{Relationship between plateau activity and discharge initiation}

Microelectrode recordings have shown that increasing the stimulus strength above threshold shortens the latency for discharge initiation (Hoffman and Haberly, 1989), and imaging techniques confirmed this (Fig. 5A) (Demir et al., 1998). Furthermore, as the stimulus strength increased above threshold, the amplitude of the plateau event increased (Fig. 5A). Thus, as noted above for subthreshold responses, plateau activity continued to increase in a graded manner as the strength of a suprathreshold stimulus was increased. The shortening of discharge latency and increase in plateau activity amplitude occurred in parallel. Thus, for a given stimulus intensity a large plateau amplitude was associated with a short discharge latency in both induced (Fig. $5 B$ ) and disinhibited (Fig. 5C) slices. Figure 5, $B$ and $C$, shows that the plateau activity amplitude associated with long latencies (achieved with nearthreshold stimulus currents) was $25 \%$ of the discharge amplitude for both models, as noted above. The limiting values at short latencies (stronger stimuli) were also similar, falling in the range of $40-50 \%$ (Fig. 5B,C). (For a given latency in the same slice the amplitude of the plateau event varied by $\sim 10 \%$ from trial to trial. The scatter in Fig. 5B,C was $>10 \%$, and this reflects variability among slices.) Evidence presented below supports the hypothesis that excitatory synapses projecting to the site of onset allow plateau activity to play a role in the genesis of an epileptiform 


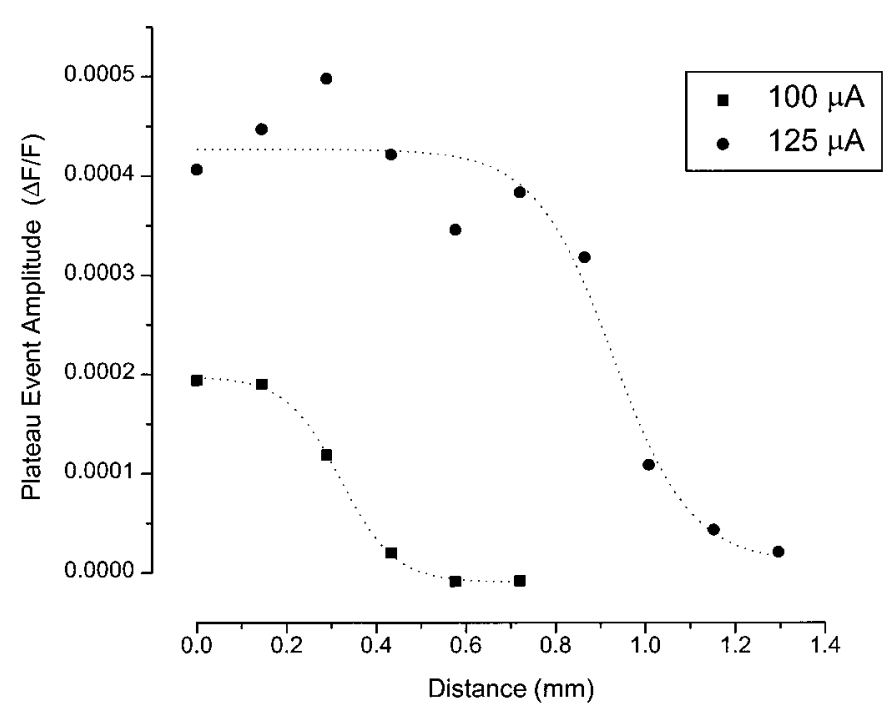

Figure 7. Plot of plateau activity amplitude versus distance. The amplitude of plateau activity was determined by measuring fluorescence from individual detectors along a line starting from the center of the blueshaded plateau activity contour of Figure $6 \mathrm{~A}$ and extending in the ventral direction (to the right in the video image). This line follows the ventrally directed (rightward) increase in size of the plateau region shown in Figure 6 . The stimulus currents were $100 \mu \mathrm{A}$ (filled squares) and $125 \mu \mathrm{A}$ (filled circles). These plots were made from the same experiment used to make Figure 6. Plots from two other experiments showed similar behavior. Sigmoidal curves were drawn to highlight the uniform amplitude within the region of plateau activity.

discharge. The shortening of latency with increasing plateau activity amplitude can be taken as consistent with this hypothesis.

Voltage imaging also showed that, as the stimulus strength increased above threshold, both the region showing onset activity and the region showing plateau activity grew. With a threshold stimulus these regions were minimal in size (Fig. 6 $\mathrm{A}$ ) and were larger with increasing suprathreshold stimuli (Fig. $6 B, C$ ). For the site of onset this growth was approximately symmetric around the small area seen with the threshold stimulus. For the site of plateau activity the growth was less symmetric and showed preferential expansion in the ventral direction (i.e., toward the right in Fig. 6). Thus with higher stimulus currents plateau activity extended through much of the extent of the En, with a little expansion into deep layer III of PC as well.

In interpreting this growth in size, it is important to check whether contours grow simply as an indirect result of increasing signal amplitude, because plateau activity amplitude increases with stimulus strength (see Fig. 5). To address this issue, we examined plots of plateau activity amplitude versus distance from the center of the blue contour in Figure $6 A$. Within the region in which it was seen, plateau activity was approximately constant in amplitude (Fig. 7; $n=3$ ). The constant amplitude within the plateau activity region indicates that the growth in the size of this zone is independent of the increase in signal amplitude. The concomitant increases in both the amplitude of plateau activity and the size of the plateau region could reflect either an increase in the number of participating cells or an increase in the depolarization of individual cells with increasing stimuli. The alternative explanation of increasing voltage spread within individual cells is less likely, because the sizes of the regions shown in Figure 7 are considerably larger than the dendritic trees of multipolar cells (Haberly, 1998). The heavy local intrinsic connections of the
En indicate that different subregions of the En have strong synaptic interactions (Behan et al., 1997; Behan and Haberly, 1999). A strong excitatory synaptic projection from neurons participating in plateau activity to neurons in the site of onset would allow increases in both the amplitude and spatial extent of plateau activity to accelerate the process of discharge initiation. Simulations of epileptiform activity indicate an intimate relationship between discharge threshold and the number of activated cells [see Traub and Miles (1991), their Fig. 6.11]. Thus, the interpretation that more cells are active during plateau activity initiated by stronger stimulus currents helps to explain the shorter latencyto-discharge onset.

\section{Blockade of plateau activity}

The observation that plateau activity precedes an epileptiform discharge raises the question of whether this activity is necessary for discharge initiation. To test this hypothesis, we blocked plateau activity in induced slices with two different pharmacological agents (see Local Drug Application, Materials and Methods). Either $\mathrm{CoCl}_{2}$, a nonselective calcium channel blocker, or kynurenic acid, a broad spectrum glutamic acid receptor antagonist, was ejected from a micropipette to reduce electrical activity at selected locations. First, the sites of onset and plateau activity were located by analyzing imaging data while the experiment was in progress. Then $\mathrm{CoCl}_{2}$ was applied to the site of plateau activity, and electrical stimulus of the same strength failed to generate a discharge $(n=12$; Fig. $8 A)$. The discharge also could be blocked by applying $\mathrm{CoCl}_{2}$ to the site of onset $(n=7$; Fig. $8 B)$, as previously reported (Hoffman and Haberly, 1991, 1996). In each case the discharge activity recovered within 2-3 min (traces 3 in Fig. $8 A, B$ ). However, when a comparable quantity of $\mathrm{CoCl}_{2}$ (see Materials and Methods) was applied to other sites in the En $(n=7)$ and adjacent neocortex $(n=8)$, epileptiform discharges still could be evoked (Fig. $8 C, D$ ). In four such experiments all four of these sites were tested in the same slice. In four additional slices we were able to test three sites. These experiments with focal application reveal the sites of plateau activity and discharge onset, as defined by imaging experiments, as the only locations at which $\mathrm{CoCl}_{2}$ can prevent the discharge from developing. Electrical activity at these sites therefore is necessary for the generation of an epileptiform discharge, and activity at other nearby sites does not play a significant role.

When applied within the plateau region, $\mathrm{CoCl}_{2}$ reduced the amplitudes of the signals there (Fig. 9A). This is relevant to the results above on the graded nature of responses at the site of plateau activity (see Figs. 4, 5). Both results are consistent with the notion that a threshold level of electrical activity must be exceeded at the plateau site to generate a discharge.

Figure 9, $B-D$, also shows signals from the plateau site to make the point that these signals were still as large as plateau activity when equal quantities of $\mathrm{CoCl}_{2}$ were applied to other nearby locations. This confirms that with our focal application procedure $\mathrm{CoCl}_{2}$ was well localized to the intended site. (This was also checked visually by observing fluorescein included in the drug solution; see Materials and Methods.) The effectiveness of drug localization was assessed further by examining responses only 375-500 $\mu \mathrm{m}$ away from the site of drug application (Fig. 10). In induced slices $\mathrm{CoCl}_{2}$ reduced the amplitude of the depolarization at the plateau site in the immediate vicinity of the $\mathrm{CoCl}_{2}$ application (see Fig. 9A). However, signals elsewhere in the plateau region only $500 \mu \mathrm{m}$ away reached the same peak amplitude as plateau 

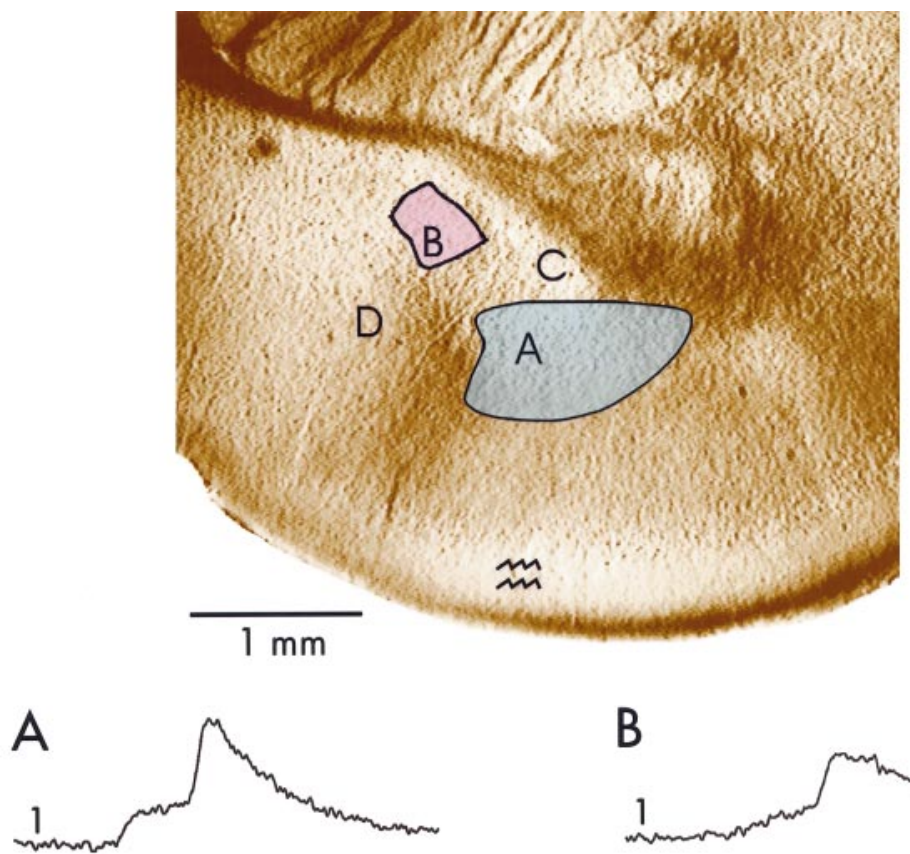

B

Figure 8. Blockade of epileptiform discharges in an induced slice by $\mathrm{CoCl}_{2}$. A solution of $10 \mathrm{mM} \mathrm{CoCl}_{2}$ was applied to the locations indicated by the letters in the video image above the traces. Fluorescence traces are displayed from each of these sites. $\mathrm{CoCl}_{2}$ was applied to the site of plateau activity (blue, $A$ ), the site of discharge onset ( pink, $B)$, the central portion of the En outside of the sites of onset and plateau activity $(C)$, and the adjacent neocortex $(D)$. The top traces (1) in each part show epileptiform discharges before $\mathrm{CoCl}_{2}$ application, and the second traces (2) show responses immediately after. In $A$ and $B$ the discharge was blocked, but not in $C$ and $D$, when the same quantity of $\mathrm{CoCl}_{2}$ was applied to each site. The third traces (3) of $A$ and $B$ show recovery 2 or $3 \mathrm{~min}$ after $\mathrm{CoCl}_{2}$ application. Stimulus currents: $A, 375 \mu \mathrm{A} ; B, 300 \mu \mathrm{A} ; C, 200 \mu \mathrm{A} ; D, 300$ $\mu \mathrm{A}$. Arrows mark the time of electrical stimulation.

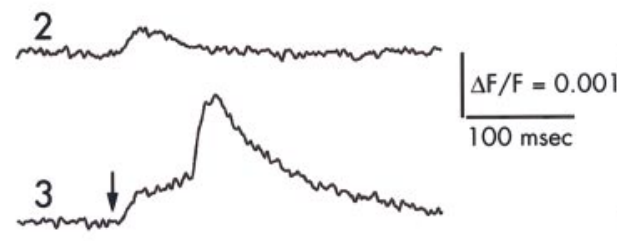

2

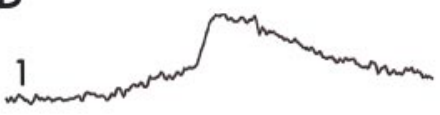
0.01
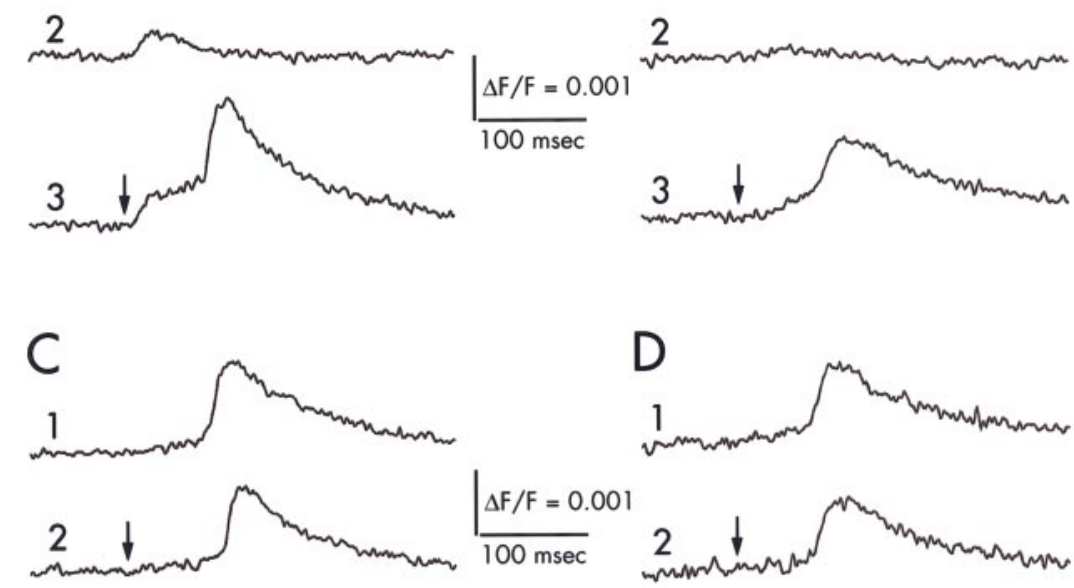

activity recorded without $\mathrm{CoCl}_{2}$ (Fig. 10A). Likewise, kynurenic acid reduced the depolarization at the plateau site at which it was applied, but signals outside the plateau region only $375 \mu \mathrm{m}$ away (representing local responses) had the same peak amplitude as those seen before kynurenic acid application (Fig. 10B).

As noted above in Figure $8 B, \mathrm{CoCl}_{2}$ blocked discharges when applied to the site of onset. Given the effectiveness of drug localization established above, this blockade is unlikely to result from the spread of $\mathrm{CoCl}_{2}$ from the onset site to the plateau site. Thus, like plateau activity, onset activity also is required for discharge generation. Although the onset site and plateau site are well separated, $\mathrm{CoCl}_{2}$ application to the site of onset produced a significant change in the depolarizations seen at the plateau site. These depolarizations were as large as those associated with plateau activity before $\mathrm{CoCl}_{2}$ application, but they were no longer sustained. Instead, they decayed smoothly to baseline (see Fig. $9 B$ ). This suggests that the sustained depolarization that characterizes plateau activity depends on feedback from the site of onset. The same observation was made with kynurenic acid (Fig. $11 B$, inset); this point will be considered further in Discussion.

As a second pharmacological test of the role of plateau activity, kynurenic acid was applied in the same way. Because kynurenic acid is a broad-spectrum excitatory amino acid receptor antagonist, this experiment tests the role of excitatory synaptic transmission at each site. Like $\mathrm{CoCl}_{2}$, kynurenic acid only blocked discharges when it was applied to the sites of plateau and onset activity (Fig. 11A,B), but not to other sites such as elsewhere in the En (Fig. 11C) or adjacent neocortex (Fig. 11D). Kynurenic acid was tested in five induced slices, with four or five sites tested in each slice, and in each experiment the results were the same as those shown in Figure 11. Furthermore, the amplitude of the signal in the plateau region was reduced only when kynurenic acid was applied at that site (Fig. 11 A, inset), rather than other nearby sites (Fig. $11 B-D$, insets). This pattern of blockade matches that obtained with $\mathrm{CoCl}_{2}$ (see Fig. 8), supporting the same conclusions that (1) both onset activity and plateau activity are necessary for the generation of epileptiform discharges, (2) the level of activity at the plateau site is critical for discharge generation, (3) blockade by reducing activity at the site of onset occurs without reducing the peak amplitude of graded responses at the site of plateau activity, and (4) sustained depolarization at the site of plateau activity depends on feedback from the site of onset. Further, as noted above with $\mathrm{CoCl}_{2}$, the qualitatively different outcomes with different sites of kynurenic acid application con- 

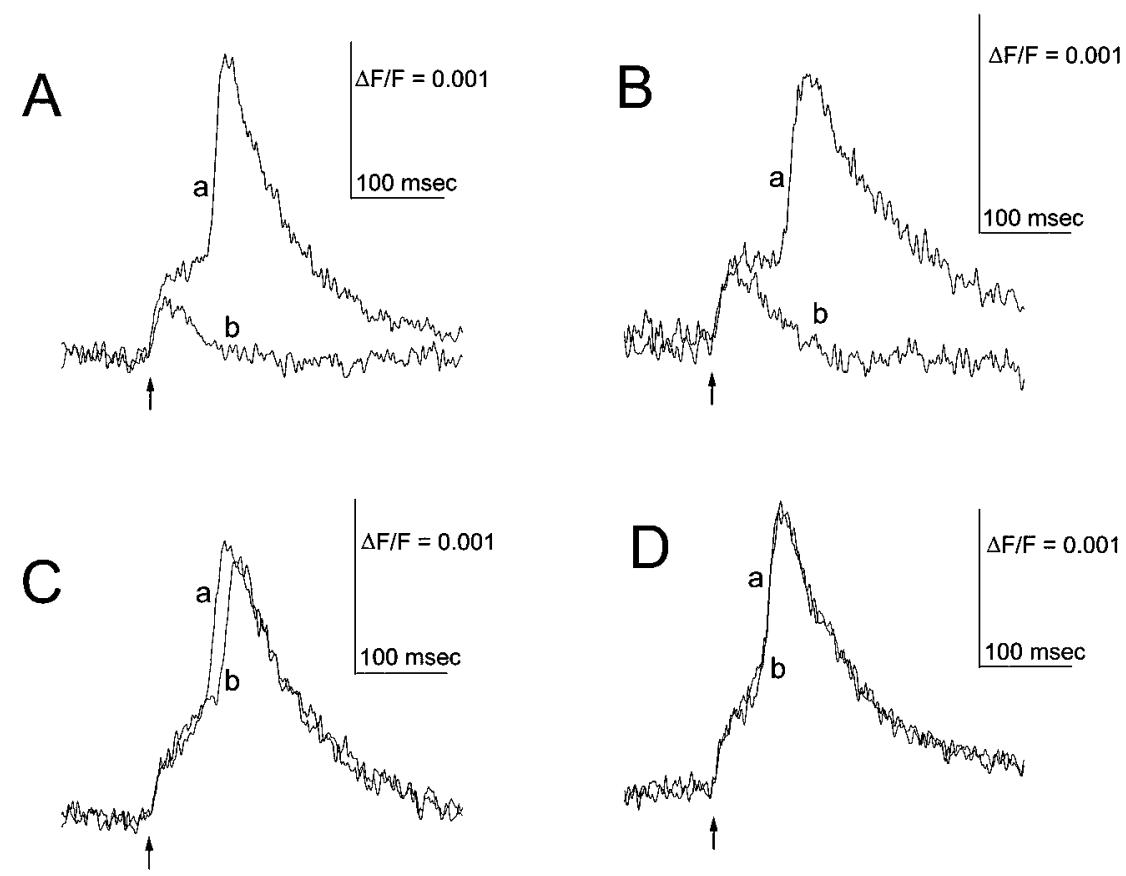

Figure 9. Fluorescence traces from the site of plateau activity. Traces were taken from the same experiment as Figure 8, with $\mathrm{CoCl}_{2}$ applied to each of the different sites indicated in the video image of Figure 8 ( $a$, before $\mathrm{CoCl}_{2}$ application; $b$, immediately after). $A, \mathrm{CoCl}_{2}$ at the site of plateau activity reduced the amplitude of the depolarization at that site and blocked the epileptiform discharge. $B, \mathrm{CoCl}_{2}$ at the site of onset blocked the epileptiform discharge but had little effect on the amplitude of the signal at the site of plateau activity. $C, D$, $\mathrm{CoCl}_{2}$ at two other sites had no effect on the level of plateau activity and did not block the epileptiform discharge.

\section{A: $\mathrm{CoCl}_{2}$}

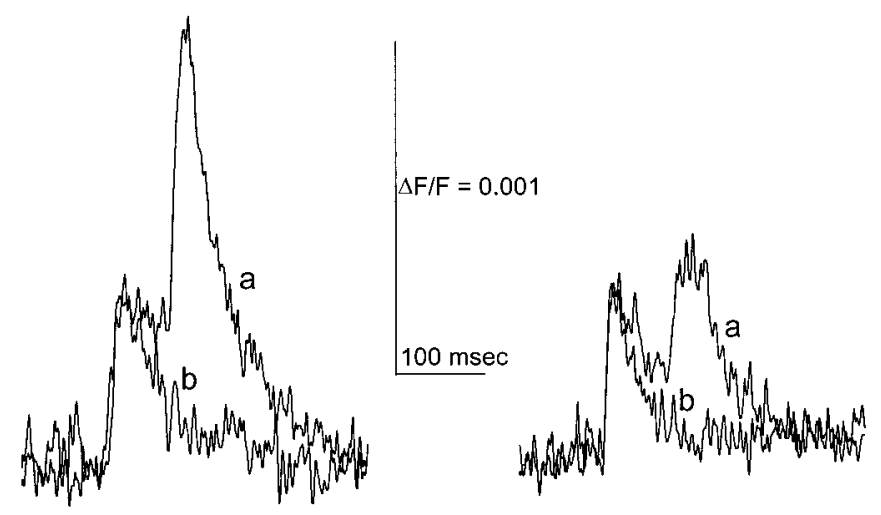

Figure 10. Control experiments for spread of $\mathrm{CoCl}_{2}$ and kynurenic acid. Signals at small distances from sites of $\mathrm{CoCl}_{2}$ and kynurenic acid application show no direct effects of these reagents. These agents were each applied to the site of plateau activity, where they reduced the amplitude of the signal locally and blocked the epileptiform discharge ( $a$, before drug application; $b$, immediately after). $A$, A trace $500 \mu \mathrm{m}$ away from the site of $\mathrm{CoCl}_{2}$ application shows that the amplitude of a signal within the plateau region was not reduced. $B$, A trace $375 \mu \mathrm{m}$ from the site of kynurenic acid application shows that the local response to stimulus outside the plateau region was not reduced. The $\mathrm{CoCl}_{2}$ data were taken from the experiment used to make Figure 8, and the kynurenic acid data were taken from the experiment used to make Figure 11.

firm the effective localization of the drug when applied by this method. These experiments with kynurenic acid confirm the important result obtained with $\mathrm{CoCl}_{2}$ that the two forms of latent electrical activity described here, plateau activity and onset activity, are both necessary for discharge generation. Electrical activity at other sites is not. Further, the experiments with this glutamic acid receptor antagonist make the additional point that glutamatergic synaptic transmission at both sites is essential for discharge generation.

\section{DISCUSSION}

This study describes a localized depolarization with a plateaulike time course that occurs during the latent period preceding epileptiform discharges. Plateau activity is seen most clearly in response to threshold electrical stimulation, because the longer latency to discharge reveals the sustained time course and plateau-like shape more clearly. Previous studies in the hippocampal slice have suggested that electrical activity increases slowly during the latent period and is distributed broadly over a large area (Traub and Miles, 1991). In slices of PC, electrical activity has been described during the latent period in the form of an accelerating build-up of extracellularly recorded multiunit spiking leading directly to a discharge (Hoffman and Haberly, 1993). With an amplitude that remains approximately constant throughout the latent period, plateau activity differs strikingly in its temporal character from the ramp-like depolarization (see Fig. 3C, trace 2) (Demir et al., 1998) and accelerating multiunit spiking (Hoffman and Haberly, 1993) at the site of onset in PC, as well as comparable activity in hippocampus (Traub and Miles, 1991).

Sustained plateau activity and ramp-like onset activity were localized in different parts of the En and adjacent deep regions. In both disinhibited and induced intermediate slices, voltage imaging confirmed the previous finding that discharges originated in the dorsal-most portion of the En (Hoffman and Haberly, 1993; Demir et al., 1998). With stimulus strengths near the threshold for discharge generation, plateau activity was seen away from the site of onset at a location that included a more superficial part of the En and adjacent deep part of layer III of the overlying PC. This site has not been identified previously as having a special role in the generation of epileptiform discharges. The distinctness of this location from the site of discharge onset suggests that the generation of an epileptiform discharge in slices of PC depends on two spatially distinct neuronal circuits. A schematic of these two circuits is shown in Figure 12 to serve as a guide in this discussion.

The two circuits depicted in Figure 12 give rise to two temporal phases in the generation of an epileptiform discharge. Plateau 

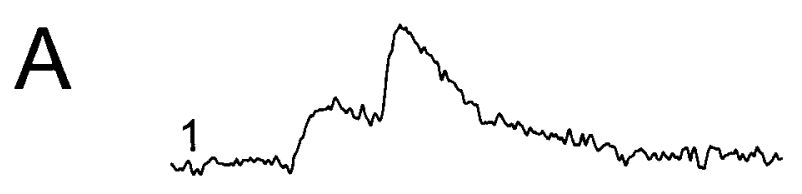

B
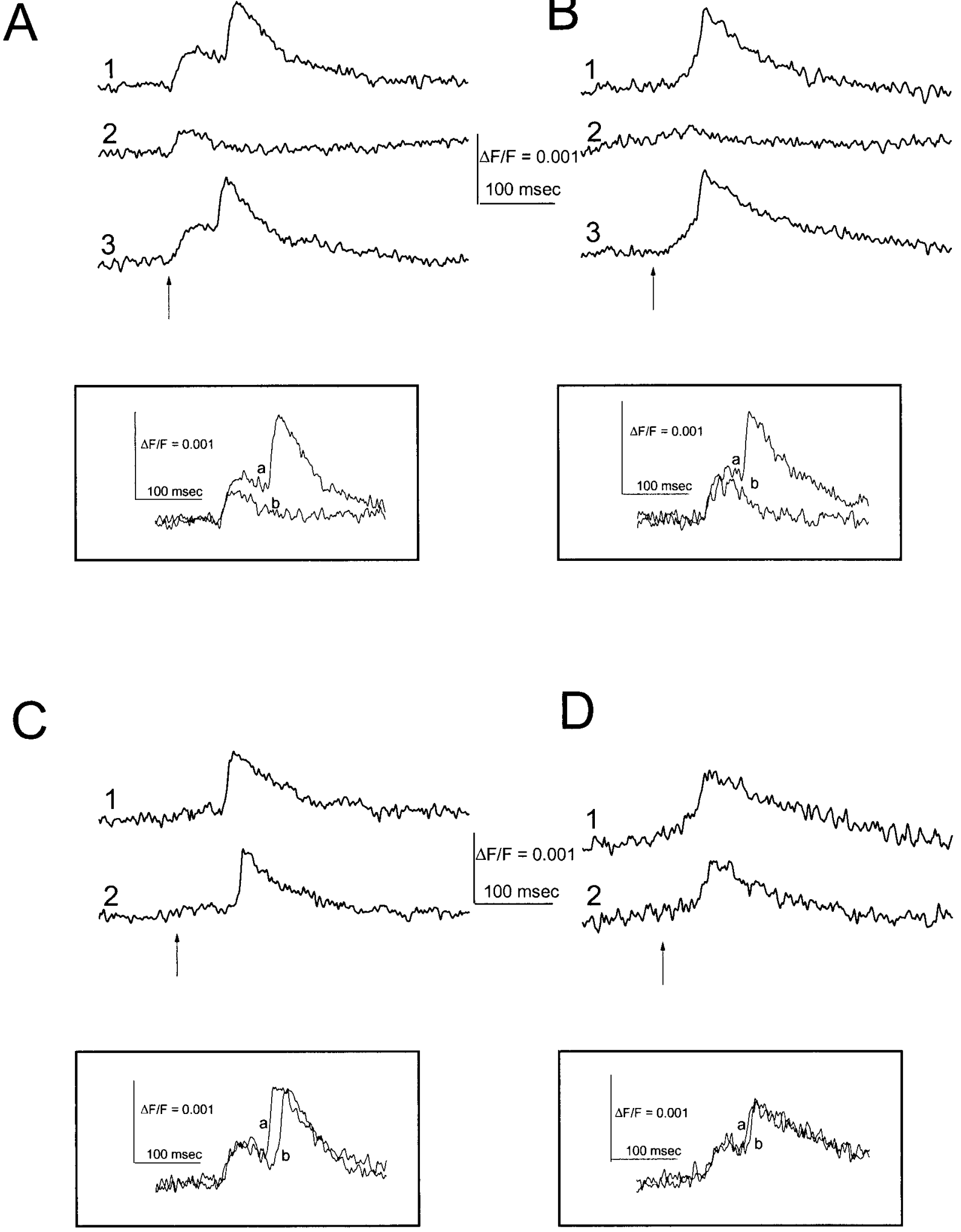

Figure 11. Blockade of epileptiform activity by kynurenic acid. Local application of $5 \mathrm{~mm}$ kynurenic acid tested the role of excitatory synaptic transmission at various locations. $A-D$ refer to approximately the same sites indicated in Figure 8 . Thus, kynurenic acid blocked discharges when applied to the site of plateau activity $(A)$ and the site of discharge onset $(B)$, but injection of the same quantity of kynurenic acid failed to block discharges when applied to another site in the En $(C)$ or to the neighboring neocortex $(D)$. The top traces $(1)$ indicate discharges before kynurenic acid application, and the second traces (2) show responses to the same electrical stimulus immediately after. In $A$ and $B$ a third trace (3) shows recovery 2 min after kynurenic acid application. In each panel the traces are shown at the site of kynurenic acid application. Arrows mark the time of electrical stimulation. All insets show fluorescence traces from the site of plateau activity (site $A$ in Fig. 8), with kynurenic acid applied to the sites indicated by the letters $A-D$ in Figure 8 ( $a$, before drug application; $b$, immediately after). Stimulus currents: $A, 250 \mu \mathrm{A} ; B, 175 \mu \mathrm{A} ; C, 200 \mu \mathrm{A} ; D, 175 \mu \mathrm{A}$. 


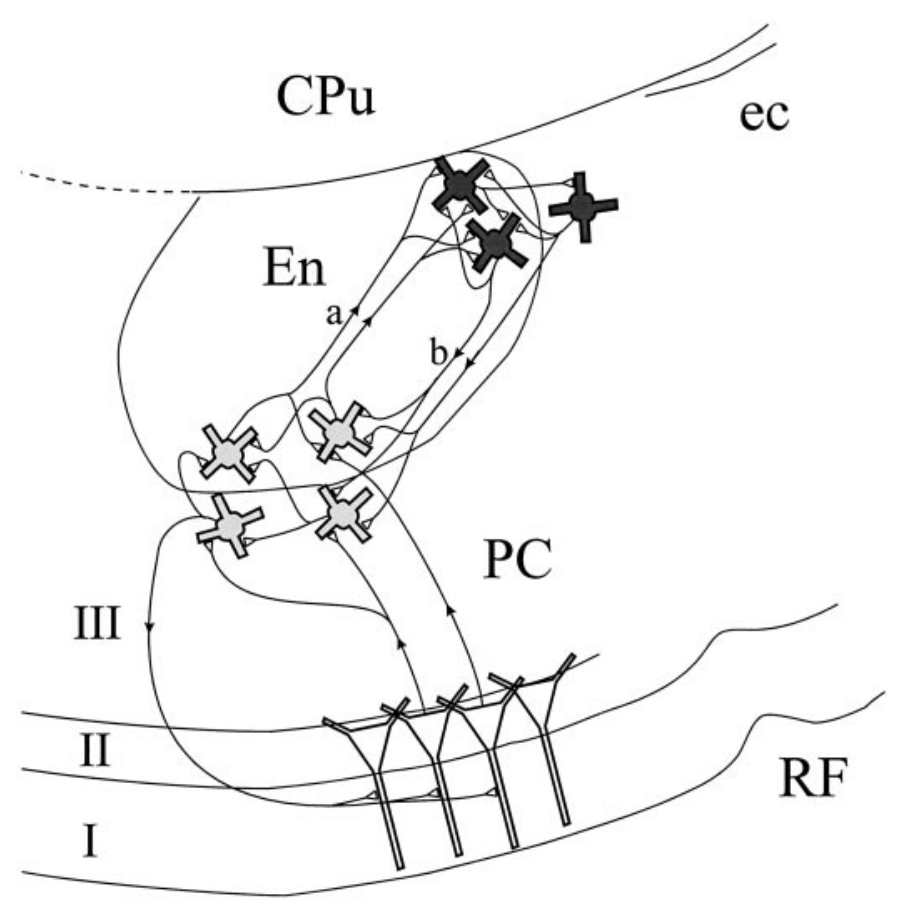

Figure 12. A minimal circuit diagram that is based on known features of PC anatomy provides a guide to the discussion of plateau activity. The structures and labels are as in Figure 1. Deep multipolar cells (windmills) concentrated in the En are connected reciprocally with layer II pyramidal cells (triangles). A suprathreshold stimulus anywhere in the PC or En activates a cluster of multipolar cells at the border of the En with deep layer III (light gray). These multipolar cells sustain plateau activity, with the aid of reciprocal excitation within this population, and project to other multipolar cells $(a)$ at the site of discharge onset (dark gray). Excitatory feedback from the site of onset to the site of plateau activity $(b)$ may contribute to sustaining plateau activity. As plateau activity continues, onset activity accelerates. The excitatory drive from the site of plateau activity to the site of onset also may be amplified by local reciprocal excitation (between the dark gray cells) to produce ramp-like onset activity, culminating in an epileptiform discharge.

activity begins a few milliseconds after stimulation, and onset activity begins $\sim 10-50 \mathrm{msec}$ later (see Fig. 3C, bottom inset). Furthermore, plateau activity remains relatively constant throughout the latent period, whereas activity at the site of onset increases rapidly, culminating in discharge onset. This temporal relationship between plateau activity and onset activity points toward a sequential process in which the electrical stimulus initiates plateau activity and plateau activity initiates the actual discharge.

The blockade produced by kynurenic acid indicates that both plateau and onset activity have a requirement for glutamatemediated excitatory synaptic transmission. The En contains a dense plexus of excitatory connections that could serve in this capacity (Hoffman and Haberly, 1993; Behan et al., 1997; Behan and Haberly, 1999). At both of these sites the requirement for synaptic excitation is likely to reflect the importance of synaptically mediated positive feedback, as proposed in a number of other studies (Ayala et al., 1973; Johnston and Brown, 1986; Wong et al., 1986; Traub and Miles, 1991; Hoffman and Haberly, 1993). Figure 12 illustrates this with strongly interconnected excitatory networks of multipolar cells at the sites of onset (dark gray cells) and plateau activity (light gray cells). Excitatory synaptic transmission at the site of plateau activity could play a role in either initiating or sustaining plateau activity. Similarly, at the site of onset, excitatory synaptic transmission can initiate the build-up with a drive from the site of plateau activity (Fig. 12, fibers labeled $a$ ) and accelerate the build-up through recurrent excitation. Moreover, the capacity of these synapses for shortterm forms of plasticity (Zucker, 1989) could be very important in controlling the time course of these depolarizations.

Plateau activity has a number of interesting properties for which cellular hypotheses must account. (1) It shows an approximately constant amplitude and can last up to $150 \mathrm{msec}$. (2) It always occurs in combination with an epileptiform discharge. (3) It depends on activity at the site of onset. (4) It is restricted spatially. (5) Depolarization at the site of plateau activity must exceed a critical level (a threshold) to generate an epileptiform discharge. One possible hypothesis for plateau activity is that diand polysynaptic activity is enhanced under epileptiform conditions. Such synaptic responses have been described in deep cells of the PC and are seen with variable delays after afferent and association fiber stimulation (Tseng and Haberly, 1989a). It is unlikely that asynchronous spike activity plays a role, because intracellular recordings during the latent period from neurons in deep PC show similar sustained depolarizations with little superimposed spike activity (Hoffman and Haberly, 1993). Slow regenerative potentials may play a role, and these could result either from the activation of $\mathrm{Ca}^{2+}$ channels (Llinás and Sugimori, 1980b; Tseng and Haberly, 1989b; Huguenard, 1996) or persistent $\mathrm{Na}^{+}$channels (Llinás and Sugimori, 1980b; Crill, 1996; Domroese and Haberly, 1996). However, the fact that sustained activity with a plateau-like time course never occurred in isolation from an epileptiform discharge argues that it cannot be a sum of plateau-like regenerative potentials occurring independently in many cells. Instead, it must require some form of collective circuit behavior, such as excitatory feedback from the site of onset (Fig. 12 , fibers labeled $b$ ). This form of feedback is the most plausible explanation for the observation that a sustained depolarization at the site of plateau activity could never be seen in the absence of an epileptiform discharge. Thus, blockade of discharges with either $\mathrm{CoCl}_{2}$ (see Fig. 8B) or kynurenic acid (see Fig. 11B) at the site of onset resulted in a response at the site of plateau activity that was as large as plateau activity but that decayed smoothly to baseline.

The spatial confinement of plateau activity may reflect the restricted distribution of a specialized class of voltage-gated or ligand-gated channels. Pyramidal cells and deep multipolar cells exhibit different physiological properties (Tseng and Haberly, 1989b). In particular, the deep multipolar cells have a smaller amplitude transient $\mathrm{K}^{+}$current than layer II pyramidal cells, and these deep cell $\mathrm{K}^{+}$channels have properties that enhance neuronal excitability (Banks et al., 1996). In addition, in guinea pig PC T-type $\mathrm{Ca}^{2+}$ channels have a higher abundance in layers III and En than layer II (Magistretti and de Curtis, 1998). However, the characterization of channel properties within specialized regions as small as those revealed in the present study has not yet been reported. Immunocytochemical techniques currently are being used to probe the distribution of various markers in the PC (Domroese et al., 1997). Preliminary results from these investigations have shown that calretinin and neuropeptide $\mathrm{Y}$ have distinct distributions in and around the En. The staining pattern of calretinin-immunoreactive processes parallels the site of onset (Demir et al., 1998). In contrast, neuropeptide Y immunoreactivity is highest in a region similar to the site of plateau activity found here. Because neuropeptide Y suppresses epileptiform activity in hippocampal slices (Klapstein and Colmers, 1997), a 
reduction in the activity of these cells in our models could account for the spatial distribution of plateau activity. Finally, it is also possible that an inhibitory field of interneurons surrounding the site of plateau activity accounts for some of the observed confinement. Anatomical tracing studies of the projections of inhibitory interneurons from this location may help to address this issue.

The finding that blockade of either plateau activity or onset activity blocks discharges raises the possibility that drugs can be developed to block these forms of electrical activity. Such drugs could be useful in the treatment of certain forms of epilepsy. This underscores the importance of determining the specific molecular and cellular properties that enable the En to generate the forms of circuit activity described here. To this purpose the PC slice could serve as a useful screening system in the search for drugs with a selective blockade of plateau activity or onset activity. The present results thus provide additional support for the use of this in vitro system as an experimental model of epilepsy. The unique combination of spatially restricted circuits that generate plateau activity and discharge onset, together with the pathways to conduct discharges to many other cortical structures (Haberly, 1998; Behan and Haberly, 1999), all could contribute to the importance of the piriform region in epileptogenesis.

\section{REFERENCES}

Ayala GF, Dichter M, Gumnit RJ, Matsumoto H, Spencer WA (1973) Genesis of epileptic interictal spikes. New knowledge of cortical feedback systems suggests a neurophysiological explanation of brief paroxysms. Brain Res 52:1-17.

Banks MI, Haberly LB, Jackson MB (1996) Layer-specific properties of the transient K-current $\left(I_{\mathrm{a}}\right)$ in piriform cortex. J Neurosci 16:3862-3876.

Behan M, Haberly LB (1999) Intrinsic and efferent connections of the endopiriform nucleus in rat. J Comp Neurol, in press.

Behan M, Sachdev P, Haberly LB (1997) Intrinsic and efferent connections of the endopiriform nucleus that may contribute to epileptogenesis. Soc Neurosci Abstr 23:317.8.

Burwell RD, Witter MP, Amaral DG (1995) The perirhinal and postrhinal cortices of the rat: a review of the neuroanatomical literature and comparison with findings from the monkey brain. Hippocampus 5:390-408

Cechetto DF, Saper CB (1987) Evidence for a viscerotopic sensory representation in the cortex and thalamus in the rat. J Comp Neurol 262:27-45.

Chien C-B, Pine J (1991) Voltage-sensitive dye recording of action potentials and synaptic potentials from sympathetic microcultures. Biophys J 60:697-711.

Colom LV, Saggau P (1994) Spontaneous interictal-like activity originates in multiple areas of the CA2-CA3 region of hippocampal slices. J Neurophysiol 71:1574-1585.

Crill WE (1996) Persistent sodium current in mammalian central neurons. Annu Rev Physiol 58:349-362.

Demir R, Haberly LB, Jackson MB (1997) Activity at two discreet sites in deep piriform cortex is required for epileptiform discharge. Soc Neurosci Abstr 23:836.9.

Demir R, Haberly LB, Jackson MB (1998) Voltage imaging of epileptiform activity in slices from rat piriform cortex: onset and propagation. J Neurophysiol 80:2723-2742.

Domroese ME, Haberly LB (1996) Dual origin of slow regenerative potentials in the endopiriform nucleus. Soc Neurosci Abstr 22:823.15.
Domroese ME, Behan M, Haberly LB (1997) Interneurons in the endopiriform nucleus and deep piriform cortex that may be involved in epileptogenesis. Soc Neurosci Abstr 23:316.14.

Grinvald A, Frostig RD, Lieke E, Hildesheim R (1988) Optical imaging of neuronal activity. Physiol Rev 68:1285-1366.

Haberly LB (1998) Olfactory Cortex. In: The synaptic organization of the brain (Shepherd GM, ed), pp 377-416. New York: Oxford UP.

Hoffman WH, Haberly LB (1989) Bursting induces persistent all-ornone EPSPs by an NMDA-dependent process in piriform cortex. J Neurosci 9:206-215.

Hoffman WH, Haberly LB (1991) Bursting-induced epileptiform EPSPs in slices of piriform cortex are generated by deep cells. J Neurosci 11:2021-2031.

Hoffman WH, Haberly LB (1993) Role of synaptic excitation in the generation of bursting-induced epileptiform potentials in the endopiriform nucleus and piriform cortex. J Neurophysiol 70:2550-2561.

Hoffman WH, Haberly LB (1996) Kindling-induced epileptiform potentials in piriform cortex slices originate in the underlying endopiriform nucleus. J Neurophysiol 76:1430-1438.

Huguenard JR (1996) Low-threshold calcium currents in central nervous system neurons. Annu Rev Physiol 58:329-348.

Jackson MB, Scharfman HE (1996) Positive feedback from hilar mossy cells to granule cells in the dentate gyrus revealed by voltage-sensitive dye and microelectrode recording. J Neurophysiol 76:601-616.

Johnston D, Brown TH (1986) Control theory applied to neural networks illuminates synaptic basis of interictal epileptiform activity. Adv Neurol 44:263-274.

Klapstein GJ, Colmers WF (1997) Neuropeptide Y suppresses epileptiform activity in rat hippocampus in vitro. J Neurophysiol 78:1651-1661.

Llinás R, Sugimori M (1980a) Electrophysiological properties of in vitro Purkinje cell somata in mammalian cerebellar slices. J Physiol (Lond) 305:171-195.

Llinás R, Sugimori M (1980b) Electrophysiological properties of in vitro Purkinje cell dendrites in mammalian cerebellar slices. J Physiol (Lond) 305:197-213.

Magistretti J, de Curtis M (1998) Low-voltage-activated T-type calcium currents are differently expressed in superficial and deep layers of guinea pig piriform cortex. J Neurophysiol 79:808-816.

McIntyre DC, Kelly ME, Staines WA (1996) Efferent projections of the anterior perirhinal cortex of the rat. J Comp Neurol 369:302-318.

Paxinos G, Watson C (1998) The rat brain in stereotaxic coordinates, Ed 4. New York: Academic.

Stasheff SF, Anderson WW, Clark S, Wilson WA (1989) NMDA antagonists differentiate epileptogenesis from seizure expression in an in vitro model. Science 245:648-651.

Traub RD, Miles R (1991) Neuronal networks of the hippocampus. New York: Cambridge UP.

Traub RD, Miles R, Wong RKS (1989) Model of the origin of rhythmic population oscillations in the hippocampal slice. Science 243:1319-1325.

Tseng GF, Haberly LB (1989a) Deep neurons in piriform cortex. I. Morphology and synaptically evoked responses including a unique high-amplitude paired shock facilitation. J Neurophysiol 62:369-385.

Tseng GF, Haberly LB (1989b) Deep neurons in piriform cortex. II. Membrane properties that underlie unusual synaptic responses. J Neurophysiol 62:386-400.

Wong RKS, Traub RD, Miles R (1986) Cellular basis of neuronal synchrony in epilepsy. In: Basic mechanisms of the epilepsies (DelgadoEscueta AV, Ward AA, Woodbury DM, Porter RJ, eds), pp 583-592. New York: Raven.

Wu JY, Cohen LB (1993) Fast multisite optical measurements of membrane potential. In: Fluorescent and luminescent probes for biological activity (Mason WT, ed), pp 389-404. London: Academic.

Zucker RS (1989) Short-term synaptic plasticity. Annu Rev Neurosci 12:13-31. 